\title{
THE TAYLOR AND OTHER METHODS OF SUMMABILITY ${ }^{1}$
}

DONALD E. MOSER

1. Introduction. The Taylor methods of summability, for which the defining matrices are

$$
T(r) \equiv T_{n k}(r)=(1-r)^{n+1} C_{k, n} r^{k-n}, r \neq 1,
$$

have been proved in [2] to be regular if and only if $0<r<1$. The methods $S(q)$, with matrices

$$
S(q) \equiv S_{n k}(q)=(1-q)^{n+1} C_{n+k, n} q^{k}, \quad q \neq 1,
$$

are regular if and only if $0<q<1$ as proved in [3] and [4]. The question of inclusion of $T(r)$ and $S(q)$ was discussed by Schoonmaker [4].

In $\S \S 2$ and 3 it will be shown that both $T(r)$ and $S(q)$ include the binary method $Y$, [5], and that neither $T(r)$ nor $S(q)$ includes the Cesàro method $C(1)$ or the Abel method $A$. $\$ 4$ consists of the determination of domains in which the geometric series $\sum z^{n}$ and the power series $\sum a_{n} z^{n}$ are summable by the iterated product method $B \cdot T(r)$, where $B$ denotes the Borel exponential method. A summary of similar results for the product method $B \cdot S(q)$ comprises $\S 5$. The author is indebted to his referee for his several helpful comments and especially for his suggestions which led to simpler proofs of Theorems 3,6 and 7 .

2. Methods $T(r)$ and $S(q)$ compared with $Y$. Consider a $Y$-summable sequence $\left\{s_{k}\right\}$. Then the sequence $\left\{y_{k}\right\}$ with $y_{k}=\left(s_{k-1}+s_{k}\right) / 2$, $k=0,1,2, \cdots$ is convergent and it follows that $s_{k}=2 \sum_{i=0}^{\boldsymbol{k}}(-1)^{k+i} y_{i}$, $k=0,1,2, \cdots$. We use these facts in the proof of

Theorem 1. If $0<r<1$, then $T(r) \supset Y$.

Proof. The $T(r)$ transform of $\left\{s_{k}\right\}$ is $\left\{\tau_{n}\right\}$, where

$$
\begin{aligned}
\tau_{n} & =2(1-r)^{n+1} \sum_{k=n}^{\infty} C_{k, n} r^{k-n} \sum_{i=0}^{k}(-1)^{k+i} y_{i} \\
& =2(1-r)^{n+1} \sum_{k=0}^{\infty} \sum_{i=0}^{n+k} C_{n+k, n} r^{k}(-1)^{n+k+i} y_{i},
\end{aligned}
$$

so

Received by the editors July 17, 1957 and, in revised form, March 30, 1959.

${ }^{1}$ Much of this work was done as a part of the author's doctoral dissertation at the University of Pittsburgh under the guidance of Dr. George Laush. 


$$
\begin{array}{r}
=2(1-r)^{n+1}\left[\sum_{i=0}^{n-1}(-1)^{n+i} y_{i} \frac{1}{(1+r)^{n+i}}\right. \\
\left.+\sum_{i=n}^{\infty}(-1)^{n+i} y_{i} \sum_{k=0}^{\infty} C_{k+i, n}(-r)^{k+i-n}\right] .
\end{array}
$$

If we integrate the sum on $k$ in (3) $n$ times, sum the resulting geometric series, and differentiate $n$ times, we obtain $\tau_{n}=G\left\{y_{i}\right\}$, where the matrix $G$ is defined by

(4) $G \equiv G_{n i}=2\left(\frac{1-r}{1+r}\right)^{n+1}(-1)^{n+i}, \quad(i=0,1,2, \cdots, n-1)$, and

$$
\begin{aligned}
G=2\left(\frac{1-r}{1+r}\right)^{n+1}(-1)^{n} r^{i} \sum_{j=0}^{n}(-1)^{i} C_{i, j} & \left(\frac{1+r}{r}\right)^{j}, \\
& (i=n, n+1, \cdots) .
\end{aligned}
$$

The remainder of the proof consists of a demonstration of the fact that the matrix $G$ is regular. We show that $G$ satisfies the three conditions of the Silverman-Toeplitz theorem.

It is clear that, for each fixed value of $i, \lim _{n} G_{n i}=0$. Secondly, since $\sum_{i=0}^{\infty} G_{n i}$ is equal to $\tau_{n}$ with $y_{i}=1$ for all $i$, it follows that

$$
\begin{aligned}
\sum_{i=0}^{\infty} G_{n i} & =(1-r)^{n+1} \sum_{k=n}^{\infty} C_{k, n} r^{k-n} 2 \sum_{i=0}^{k}(-1)^{k+i} \\
& =1+(-1)^{n}\left(\frac{1-r}{1+r}\right)^{n+1}
\end{aligned}
$$

and therefore, that

$$
\lim _{n} \sum_{i=0}^{\infty} G_{n i}=1
$$

Finally, from (4) and (5) and with the aid of the inequality

$$
\sum_{j=0}^{n}\left(\frac{1+r}{r}\right)^{j} \sum_{i=n}^{\infty} C_{i, j} r^{j} \leqq \sum_{j=0}^{n}\left(\frac{1+r}{r}\right)^{j} \sum_{i=j}^{\infty} C_{i, j} r^{j},
$$

it follows that

$$
\begin{aligned}
\sum_{i=0}^{\infty}\left|G_{n i}\right| & \leqq 2\left(\frac{1-r}{1+r}\right)^{n+1}\left[\sum_{i=0}^{n-1} 1^{i}+\sum_{i=n}^{\infty} r^{i} \sum_{j=0}^{n} C_{i, j}\left(\frac{1+r}{r}\right)^{j}\right] \\
& <2\left(n-\frac{1}{2 r}\right)\left(\frac{1-r}{1+r}\right)^{n+1}+\frac{1}{r}
\end{aligned}
$$


and, therefore, that

$$
\lim \sup \sum_{i=0}^{\infty}\left|G_{n i}\right| \leqq \frac{1}{r} .
$$

Thus the sufficient conditions of the Silverman-Toeplitz theorem are satisfied, $G$ is a regular matrix, and the proof is complete.

Because of the similarity of the matrices defined in (1) and (2), the same pattern of proof may be used to establish

Theorem 2. If $0<q<1$, then $S(q) \supset Y$.

The details of the proof are omitted.

3. $T(r)$ and $S(q)$ compared with $C(k)$ and $A$. It is well known that $A \supset C(k) \supset C(1)$ when $k>1$. We therefore consider the question of inclusion of $C(1)$ by $T(r)$ and prove

THEOREM 3. If $0<r<1$, then $T(r) D C(1)$.

Proof. Let $\left\{s_{k}\right\}$ be a sequence which is summable $C(1)$ so that $\left\{c_{k}\right\}$ is convergent when $c_{k}=\left(s_{0}+s_{1}+\cdots+s_{k}\right) /(k+1)$. Then $s_{k}=(k+1) c_{k}-k c_{k-1}$. With $r$ fixed between 0 and 1 , we apply the $T(r)$ matrix to $\left\{s_{k}\right\}$ to obtain

$$
\tau_{n}=\sum_{k=n}^{\infty}(1-r)^{n+1} C_{k, n} r^{k-n}\left[(k+1) c_{k}-k c_{k-1}\right]=H\left\{c_{k}\right\}
$$

where, when $k \geqq n$,

$$
H_{n k}=(1-r)^{n+1} C_{k, n} r^{k-n}[(k+1-n)-r(k+1)] \frac{k+1}{k+1-n} .
$$

Since the last factor is always 1 or more, we see that $\left|H_{n k}\right| \geqq a_{n k}$ where

$$
a_{n k}=(1-r)^{n+1} C_{k, n} r^{k-n}|(k+1-n)-r(k+1)| .
$$

We find that

$$
(1-r)^{-1} \sum_{k=n}^{\infty} a_{n k}=(1-r)^{n+1} \sum_{k=0}^{\infty} C_{k+n, k} r^{k}\left|(k+1)-\lambda_{n}\right|,
$$

where $\lambda=r /(1-r)$ and hence $\lambda>0$. Let $M$ be a positive constant and, for each $n$, let $E(n)$ denote the finite set of values of $k$ for which $|k+1-\lambda n|<M$. Then 


$$
\begin{aligned}
& (1-r)^{-1} \sum_{k=n}^{\infty} a_{n k} \\
& >(1-r)^{n+1} \sum_{k=0}^{\infty} C_{n+k, n} r^{k} M-(1-r)^{n+1} \sum_{k \in E(n)} C_{n+k, n} r^{k} M \\
& \quad=M-(1-r)^{n+1} \cdot M \cdot \sum_{k \in E(n)} C_{n+k, n} r^{k} .
\end{aligned}
$$

By use of Stirling's formula and the fact that there are no more than $2 M+2$ members of $E(n)$, we can show that

$$
\lim _{n \rightarrow \infty}(1-r)^{n+1} M \sum_{k \in E(n)} C_{n+k, n} r^{k}=0
$$

hence,

$$
\lim _{n \rightarrow \infty} \sum_{k=n}^{\infty} a_{n k}>M(1-r), M \text { arbitrary. }
$$

It follows that

$$
\lim _{n \rightarrow \infty} \sum_{k=n}^{\infty}\left|H_{n k}\right|=\infty,
$$

and so $H$ is not a regular matrix and the conclusion of Theorem 3 follows.

It is obvious, in light of the remark at the beginning of this section, that $T(r) D A$ and $T(r) D C(k)$ for each $k$.

Similar results are obtained when the question of inclusion of $C(1)$ by $S(q)$ is considered.

Theorem 4. If $0<q<1$, then $S(q) D C(1)$.

Proceeding exactly as in the proof of Theorem 3, it is easily established that, with $q$ fixed between 0 and 1 , the $S(q)$ transform of $\left\{s_{k}\right\}$ is $\sigma_{n}=V\left\{c_{k}\right\}$ where

$$
V_{n k}=(1-q)^{n+1} q^{k} C_{n+k, n}[k+1-q(n+k+1)] .
$$

We find that

$$
(1-q) \sum_{k=0}^{\infty}\left|V_{n k}\right|=(1-q)^{n+1} \sum_{k=0}^{\infty} C_{n+k, n} q^{k}|k+1-\lambda n|
$$

where $\lambda=q /(1-q)$ and observing the similarity of (7) and (6), we obtain the conclusion of Theorem 4.

It follows, as before, that $S(q) \nsupseteq A$ and $S(q) D C(k)$ for any $k$. 
4. Power series and the product method $B \cdot T(r)$. Consider the complex geometric series $\sum z^{n}$ with partial sums $s_{k}=(1-z)^{-1}\left(1-z^{k+1}\right)$, and consider the Borel exponential transform of the $T(r)$ transform of $\left\{s_{k}\right\}$. We observe that $T(r)$ is applicable to $\left\{s_{k}\right\}$ if and only if $z \neq 1$ and $z<r^{-1}$ and prove

Theorem 5. If $0<r<1$, the complex geometric series $\sum z^{n}$ is summable to $(1-z)^{-1}$ by the iterated product method $B \cdot T(r)$ for all $z$ in the region

$$
D B \cdot T(r):|z|<r^{-1},\left|z-\left(r^{-1}+1\right) / 2\right|>\left(r^{-1}-1\right) / 2 .
$$

Proof. With $z \neq 1$ and $|z|<r^{-1}$ we find that $B \cdot T(r)\left\{s_{k}\right\}$ is

$$
\begin{aligned}
F(t) & =e^{-t} \sum_{n=0}^{\infty} \frac{t^{n}}{n !}(1-r)^{n+1} \sum_{k=n}^{\infty} C_{k, n} r^{k-n}(1-z)^{-1}\left(1-z^{k+1}\right) \\
& =(1-z)^{-1}-\frac{(1-r) z}{(1-z)(1-r z)} e^{t(z-1)(1-r z)^{-1}},
\end{aligned}
$$

and the limit of $F(t)$ as $t \rightarrow \infty$ is $(1-z)^{-1}$ if $z$ lies in the region determined by $R(z-1)(1-r z)^{-1}<0$. It is a simple calculation to show that this is the second region in (8).

The nature of $D B \cdot T(r)$ suggests that it may be possible to choose $r$ so that for any preassigned $z$ in the Mittag-Leffler star associated with $\sum z^{n}$ the series will be summable $B \cdot T(r)$. That this is the case is

TheOREM 6. Let $z$ be any point of the Mittag-Leffler star $S$ associated with the series $\sum z^{n}$, and take $r=(|z-1|+1)^{-1}$. Then $\sum z^{n}$ is summable $B \cdot T(r)$.

This is a consequence of the following simple fact which involves three circles having diameters on the real axis. Each point $z$ which lies on the circle $|z-1|=r^{-1}-1$ but not on the half line $z \geqq 1$ lies inside the circle $|z|=r^{-1}$ and outside the circle $\left|z-\left(r^{-1}+1\right) / 2\right|=\left(r^{-1}-1\right) / 2$ and hence in the region $D B \cdot T(r)$.

Now consider the power series $\sum a_{n} z^{n}$ with finite radius of convergence $R$, and let $f$ denote the function obtained by analytic continuation along radial lines from the origin. Corresponding to each singularity $\xi$ of $f$, and with $r$ fixed, let $C_{\xi}(r)$ denote the set of points $z$ for which

$$
|z|<r^{-1}|\xi|, \quad\left|z / \lambda-\left(r^{-1} \xi+\xi\right) / 2\right|>\left|r^{-1} \xi-\xi\right| / 2
$$

whenever $\lambda \geqq 1$, and let $C(r)$ denote the intersection of the sets $C_{\xi}(r)$.

THEOREM 7. For each fixed $r$ satisfying $0<r<1$, the series $\sum a_{n} z^{n}$ is summable $B \cdot T(r)$ to $f(z)$ at every interior point $z$ of the region $C(r)$. 
Proof. Let $z$ be a fixed point of $C(r)$. Then $|r z|<|\xi|$ for all singularities $\xi$, and it follows that the circle $C_{1}$ with center at the origin and radius $|r z|$ is contained in the Mittag-Leffler star $S$ of $f$. Also, when $\lambda \geqq 1$,

$$
\left|z / \lambda \xi-\left(r^{-1}+1\right) / 2\right|>\left|r^{-1}-1\right| / 2
$$

and, as we see by making an inversion in which complex numbers are replaced by their reciprocals,

$$
|\lambda \xi-(z+r z) / 2|>|z-r z| / 2 \text {. }
$$

This means that, whenever $\xi$ is a singular point of $f$ and $\lambda \geqq 1$, the point $\lambda \xi$ lies outside the circle $C_{2}$ with center at $(z+r z) / 2$ and radius $|z-r z| / 2$. In other words, the circle $C_{2}$ of points $u$ for which

$$
|u-(z+r z) / 2|=|z-r z| / 2
$$

lies in $S$. This implies that we can choose a simple closed curve $\gamma$ which has finite length, which lies in $S$, and which contains both of the circles $C_{1}$ and $C_{2}$ in its interior. Since the origin is in $C_{1}$ and $z$ is on $C_{2}$, both of these points are inside $\gamma$. We may then write

$$
a_{n}=\frac{1}{2 \pi i} \int_{\gamma} \frac{f(u)}{u^{n+1}} d u
$$

and hence, for each $k$

$$
\begin{aligned}
s_{k}(z) & =\sum_{n=0}^{k} z^{n} \frac{1}{2 \pi i} \int_{\gamma} \frac{f(u)}{u^{n+1}} d u \\
& =f(z)-\frac{1}{2 \pi i} \int_{\gamma} \frac{z f(u)}{u(u-z)}\left(\frac{z}{u}\right)^{k} d u .
\end{aligned}
$$

The $B \cdot T(r)$ transform of $\left\{s_{k}\right\}$ is

$$
F(x)=f(z)-R_{z}(x)
$$

where

$$
\begin{aligned}
R_{z}(x) & =e^{-x} \sum_{n=0}^{\infty} \frac{x^{n}}{n !}(1-r)^{n+1} \sum_{k=n}^{\infty} C_{k, n} r^{k-n} \frac{1}{2 \pi i} \int_{\gamma} \frac{z f(u)}{u(u-z)}\left(\frac{z}{u}\right)^{k} d u \\
& =\frac{e^{-x}}{2 \pi i} \sum_{n=0}^{\infty} \frac{x^{n}}{n !}(1-r)^{n+1} \int_{\gamma} \frac{z f(u)}{u(u-z)}\left(\frac{z}{u}\right)^{n}\left(\frac{u}{u-r z}\right)^{n+1} d u \\
& =\frac{1-r}{2 \pi i} \int_{\gamma} \frac{z f(u)}{(u-z)(u-r z)} \exp \left(x\left(\frac{z-u}{u-r z}\right)\right) d u .
\end{aligned}
$$


We observe that $\lim _{x \rightarrow \infty} R_{z}(x)=0$ since, for all points $u$ on $\gamma, u$ is exterior to $C_{2}$ and hence $R((z-u) /(u-r z))<0$. Therefore, from (10) we have

$$
\lim _{x \rightarrow \infty} F(x)=f(z)
$$

and the proof is complete.

5. Power series and the product method $B \cdot S(q)$. As is to be expected, it is found that Theorems 5,6 and 7 also hold when the product method $B \cdot T(r)$ is replaced by $B \cdot S(q)$. The proofs are so nearly identical to those already given that they are omitted here.

\section{REFERENCES}

1. G. H. Hardy, Divergent series, Oxford, 1949.

2. G. Laush, Relations among the Weierstrass methods of summability, Cornell University doctoral dissertation, 1949.

3. W. Meyer-Konig, Untersuchungen über einige verwandte Limitierungsverfahren, Math. Z. vol. 52 (1949) pp. 257-304.

4. N. J. Schoonmaker, Inclusion relations among methods of summability, Proc. Amer. Math. Soc. vol. 7 (1956) pp. 102-108.

5. O. Szász, On the product of two summability methods, Ann. Soc. Polon. Math. vol. 25 (1952) pp. 75-84. 1952.

6. - Introduction to the theory of divergent series, University of Cincinnati,

7. P. Vermes, Series to series transformations and analytic continuation by matrix methods, Amer. J. Math. vol. 71 (1940) pp. 541-562.

UNiversity OF PITTSBURgh AND

UNIVERSITY OF MASSACHUSETTS 